\title{
Understanding the radio emission geometry of PSR B0329+54
}

\author{
R. T. Gangadhara ${ }^{1}$ and Y. Gupta ${ }^{2}$ \\ ${ }^{1}$ Indian Institute of Astrophysics, Bangalore-560034, India \\ ${ }^{2}$ National Centre for Radio Astrophysics, TIFR, \\ Pune University Campus, Pune-41100\%, India
}

\begin{abstract}
We have analyzed high-quality single pulse data of PSR B0329+54 at $325 \mathrm{MHz}$ and $606 \mathrm{MHz}$ to study the structure of the emission beam. Using the windowthreshold technique, which is suitable for detecting weak emission components, we have detected 4 additional emission components in the pulse window. Three of these are new components and the fourth is a confirmation of a recently proposed component. Hence PSR B0329+54 is now known to have 9 emission components - the highest among all known pulsars. The distribution of the pulse components around the central core component indicates that the emission beam consists of four nested cones. The asymmetry in the location of the conal components in the leading versus trailing parts of the profile is interpreted as being due to aberration and retardation in the pulsar magnetosphere. These measurements allow us to determine the precise location of the 4 conal rings of emission. We find that the successive outer cones are emitted at higher altitudes in the magnetosphere. Further, for any given cone, the emission height at the lower frequency is found to be more than that at the higher frequency. The inferred heights range from $\sim 160 \mathrm{~km}$ to $\sim 1150 \mathrm{~km}$. The set of "active" field lines, from which most of the conal radiation appears to originate, are found to be confined to a region located within $\sim 0.5$ to $\sim 0.6$ of the polar cap radius. We discuss the implications of our new findings on our understanding of the pulsar emission geometry and its impact on the emission mechanisms.
\end{abstract}

Subject headings: pulsars: individual: PSR B0329+54, emission beam structure, polar cap.

\footnotetext{
${ }^{1}$ E-mail: ganga@iiap.ernet.in

${ }^{2}$ E-mail: ygupta@ncra.tifr.res.in
} 


\section{Introduction}

Radio wave emission from pulsars is believed to originate in the open field line region of the polar cap of the neutron star. The size, shape and location of regions of radio emission in the average profiles of pulsars is thus expected to reflect the arrangement of emission regions in the pulsar magnetosphere. Pulsar average profiles exhibit a great diversity in shape, and their classification based on the number of emission components is a useful starting point to study the emission characteristics of pulsars. Rankin (1983a,b, 1990, 1993) has carried out such a detailed classification, and has come up with the conclusion that there are two kinds of emission components - core and conal - in pulsar profiles, which result from two distinct types of emission mechanisms. Further, Rankin proposes that the conal components arise from two nested hollow cones of emission, which along with a central core emission region, make up the complete pulsar emission beam. The actual profile observed for a given pulsar depends on the cut that the observer's line-of-sight makes through this emission beam. From the above work, Rankin also concludes that core radiation originates from very close to the neutron star surface whereas the conal radiation comes from regions higher up in the magnetosphere. The outer cone is postulated to originate higher up in the magnetosphere than the inner cone, but along the same set of field lines. However, there are other reports, (e.g. Gil 1991), which postulate that the core and conal emissions might originate at very similar heights in the magnetosphere.

Lyne and Manchester (1988) also confirm the difference in properties between the core and conal emissions. However, they believe that emission within the beam is patchy, i.e. the distribution of component locations within the beam is random, rather than organized in one or more hollow cones. They have come to the conclusion that a single emission mechanism can account for both the central and outer components. Further, they propose that the observations are best described by a gradual change in emission characteristics from the axial region to the outer edge of the emission beam, rather than by two distinct emission processes.

In this paper, we present the results from an analysis of emission components of PSR B0329+54. This pulsar, one of the strongest known at radio wavelengths, is an excellent laboratory for a detailed study of pulsar emission physics. The simplest radio observations of this pulsar show a profile with three clearly visible components and in the classification scheme of Rankin (1990), it is identified as a "triple (T)" profile. The central component is thought to be due to core emission and the two outer components are of the conal type. However, there has been some evidence for the presence of more than three distinct components for this pulsar. For example, Hesse (1973) has adopted five components for a study of the relative intensity variations between the different components. More recently, Kuzmin 
and Izvekova (1996), from a study of fitting Gaussians to distinct emission components in the average profile, have found that a five component model does not adequately describe this pulsar's profile. They propose a six component model and thereby question the validity of the core-cone structure of the emission beam. Hence, detection of the correct number of emission components and their distribution with respect to pulse longitude, plays a crucial part in deciding whether the pulsar emission beam is conal or patchy. For PSR B0329+54, this attempt is further complicated by the fact that this pulsar is a mode-changer, i.e. it switches between the two different modes of average profile (Lyne 1971; Hesse, Sieber and Wielebinski 1973), during which there is an appreciable change in the relative strengths and the locations of the emission components.

In this paper, we present the results from an analysis of single pulse data of PSR B0329+54, at two different frequencies. We have used the window-threshold technique, which is suitable for the detection of weak emission components in pulsar profiles (Gangadhara 2000). In section 2, we report the detection of new emission components in the profile of PSR B0329+54. In section 3 we interpret the results in terms of aberration and retardation effects in the pulsar magnetosphere and use this to estimate the emission heights and polar cap locations of the cones. Section 4 discusses the implications of our findings on the structure of the emission beam of this pulsar.

\section{Observations and Data analysis}

For our study of emission components of PSR B0329+54, we considered two data sets: the first obtained at $606 \mathrm{MHz}$ from the Lovell telescope at Jodrell Bank; and the second obtained at $325 \mathrm{MHz}$ from the GMRT (Giant Metrewave Radio Telescope) at Khodad in India. Table 1 summarizes the main observing parameters for these data sets. The details of the observing system and the calibration procedures for the Lovell telescope are identical to that used by Gould and Lyne (1998).

For the GMRT, which consists of 30 antennas each of 45 meter diameter (see Swarup et

Table 1: Single pulse observations of PSR B0329+54.

\begin{tabular}{cccccc}
\hline \hline Telescope & Date & $\begin{array}{c}\text { Frequency } \\
(\mathrm{MHz})\end{array}$ & Pulses & $\begin{array}{c}\text { Resolution } \\
(\mathrm{ms})\end{array}$ & $\begin{array}{c}\text { Bandwidth } \\
(\mathrm{MHz})\end{array}$ \\
\hline GMRT & March/April 1999 & 325 & 2100 & 0.516 & 16 \\
Lovell & 30 August 1996 & 606 & 2500 & 0.249 & 40 \\
\hline
\end{tabular}


al. (1990) for details), the data were obtained by incoherent addition of the dual polarization signals from 6 antennas. The bandwidth used was $16 \mathrm{MHz}$, divided into 256 spectral channels by the digital back-ends (see Gupta et al. (2000) for more details about the pulsar mode of operation of the GMRT). The raw data were integrated to a time resolution of 0.516 milliseconds before being recorded for off-line analysis, where the data were dedispersed and gated to obtain the single pulse data. During off-line analysis, care was taken to check the data for radio frequency interference signals and to filter out the power line frequency signals. The final 2100 pulses were collected from more than one observing session, each of which had the same observing parameters. For all the data sets from GMRT and Lovell, the profiles were aligned by defining the peak of the central component in the average profile as the location of zero pulse longitude.

The average pulse profiles obtained from the $325 \mathrm{MHz}$ and $606 \mathrm{MHz}$ data are shown in Fig. 1a \& b, respectively. For the Lovell data, the pulsar showed a mode change in the midst of the observations. In the beginning, for about 600 pulses, it was in the abnormal mode after which it switched to the normal mode. The dotted line curve in Fig. 1b shows the average profile when the pulsar was in the abnormal mode. All the profiles in Fig. 1 show 5 distinct emission components whose peaks lie in the longitude range of $-14^{\circ}$ to $10^{\circ}$. However, a study of the single pulses reveals the presence of significant emission outside these longitude ranges. For example, Fig. 2a shows a sequence of about 300 pulses from the abnormal mode duration, where occasional emission in the longitude ranges $-20^{\circ}$ to $-16^{\circ}$ and $13^{\circ}$ to $18^{\circ}$ can easily be seen. This extra emission can also be detected in the average profile made from these pulses (Fig. $2 \mathrm{~b}$ ).

Since the pulses which have emission in the longitude ranges $-20^{\circ}$ to $-16^{\circ}$ and $13^{\circ}$ to $18^{\circ}$ are less frequent, these emissions are not clearly seen in the average pulse profiles in Fig. 1. To enhance and easily detect emission from such regions, we have used a "windowthreshold" technique (Gangadhara 2000) in which we set a window in longitude domain and employ an intensity threshold to select the single pulses that go to make an average profile. We consider all those pulses which have emissions above the threshold within the window. As a result of this averaging of selected pulses, emission components within the window improve in signal to noise ratio compared to other parts of the profile and are more easily detected. Thus, for example, by setting a window over the longitude range $-20^{\circ}$ to $-16^{\circ}$, and considering all those pulses which are above a $4 \sigma$ intensity threshold (here, $\sigma$ is the rms of the intensity in the off pulse region) we obtained an average profile shown as profile number 8 in Fig. 3a \& b. This profile clearly shows an emission component (labeled component VIII in the figures) centered at about $-18^{\circ}$ for the $606 \mathrm{MHz}$ data and at about $-20^{\circ}$ for the $325 \mathrm{MHz}$ data. 
The "uniqueness in the phase location of a component peak (within error bars) with respect to the changes in the window position and threshold levels" was used as a criterion for deciding whether a given pulse component was detected. As a additional criterion, we also looked at its detection at other frequency and whether it follows the general trend of radius-to-frequency mapping followed by the known components.

By applying the technique repeatedly at different pulse longitude windows, we have determined the presence of 9 unique emission components, shown by the 9 average profiles in Fig. 3a\&b. The combined profile (Fig. 3c \& d), obtained by adding all these 9 profiles, shows the relative location of the 9 emission components of PSR B0329+54.

To test if this detection of components is unique, we have tried our window-threshold technique for choices of much broader longitude windows, such as those which enclose more than one component. In all such cases, our technique determines the same number and location of components. Furthermore, our technique detects no new components when applied to regions of longitude that are far away from the known on-pulse region. This argues that our detected components are genuine.

\section{Interpretation}

Since we have identified 8 conal components which are evenly distributed in number about the core component, our results can be interpreted in terms of a set of nested cones of emission. Figure $4 \mathrm{a}$ shows the location of the components in the form of 4 conal rings of emission around the core component, for the $606 \mathrm{MHz}$ data. In Fig. 4b we show the pulse phase extent of each cone at $325 \mathrm{MHz}$ and $606 \mathrm{MHz}$, along with the location of the nominal centre of the cone, taken to be the half-way point between the corresponding leading and trailing components. We estimate the phase location of any given component as the mean of the distribution of phases of the peaks of single pulses which are selected by our window-thresholding technique. We estimate an error on this component phase location as the standard deviation of the same distribution. The error bar on the location (zero phase) of the core component is estimated to be about $\pm 0.6^{\circ}$ at both the frequencies. We also estimated the component locations by fitting Gaussians to each component in Fig. 3a \& b, which gave the results that are same within the error bars with the method we have adopted. From the plots in Fig. 4, it is clear that the conal components are squeezed closer to the core (and to each other) in the trailing part of the profile, as compared to the leading part. This asymmetry is clearly reflected in the shift of the cone centers away from the core and towards the leading part of the profile. This effect increases as we go from the inner cones to the outer ones. Further, for the same cone, the effect is more pronounced at the lower 
frequency.

The above remarkable behavior has a simple interpretation: the radiation beams from the outer cones are progressively bent or deflected in the forward direction, i.e. the direction of rotation of the pulsar. Such a bending can be produced by aberration and retardation effects in the pulsar magnetosphere (Cordes 1978). Below, we consider in detail the emission geometry in the pulsar magnetosphere and include the effects of aberration and retardation. Using these, we show that the emission heights as well as the transverse location of associated dipolar magnetic field lines on the polar cap can be uniquely estimated for each cone, at each frequency of observation. We also consider the effect of magnetic field sweepback which can produce bending of the cones in the opposite direction. We find that, in the present context, the effect of magnetic field sweepback is negligible compared to the aberration and retardation effects.

\subsection{Emission geometry}

The geometry relevant to our current understanding of emission of radio radiation from the polar cap region of pulsar magnetospheres is illustrated in Fig. 5. We start with a righthanded coordinate system originating at the center of the star with the z-axis aligned parallel to the rotation axis $\hat{\Omega}$. In the figure, $\hat{m}$ and $\hat{n}$ represent unit vectors along the magnetic axis of the pulsar and along the line of sight to the observer and they are inclined at angles $\alpha$ and $\zeta$, respectively, to the rotation axis. The vectors $\hat{n}, \hat{m}$ and $\hat{\Omega}$ all lie in the x-z plane when the rotation phase $\phi=0$. In this geometry, we have

$$
\begin{gathered}
\hat{n}=\hat{z} \cos \zeta+\hat{x} \sin \zeta, \\
\hat{m}=\hat{z} \cos \alpha+\sin \alpha(\hat{x} \cos \phi+\hat{y} \sin \phi),
\end{gathered}
$$

where $\zeta=\alpha+\beta$, and $\beta$ is the impact angle of line-of-sight with respect to magnetic axis. The angle $\Gamma$ made by $\hat{n}$ with respect to $\hat{m}$ at any $\phi$ is then given by

$$
\cos \Gamma=\cos \alpha \cos \zeta+\sin \alpha \sin \zeta \cos \phi .
$$

Now consider the curve $\mathrm{C}$ in Fig. 5, which represents a typical dipolar magnetic field line. Let $\mathrm{P}$ represent an emission point on this field line, with spherical coordinates $(r, \theta)$ centered on the magnetic axis. Since the tangent to the field line at $\mathrm{P}$ needs to be parallel to $\hat{n}$, we can establish the following relationship between $\theta$ and $\Gamma$ :

$$
\tan \theta=-\frac{3}{2 \tan \Gamma} \pm \sqrt{2+\left(\frac{3}{2 \tan \Gamma}\right)^{2}}
$$


This equation has two roots, and the continuous physical solution switches the sign of second term on right hand side from positive to negative when $\Gamma$ changes sign from positive to negative. For the region close to the magnetic axis one can approximate Eq. (4) as $\theta \approx$ $(2 / 3) \Gamma$.

Let $\left(\mathbf{r}_{\mathrm{em}}^{\mathrm{i}}, \theta_{\mathrm{em}}^{\mathrm{i}}\right)$ be the coordinates of the emission point for the $\mathrm{i}^{\text {th }}$ cone, $(\mathrm{i}=1,2,3$ and 4 are the cone numbers). In the absence of any aberration and retardation, it is assumed that the radiation from these points is emitted tangential to the field lines. However, the aberration due to corotation causes emission beams to bend towards the azimuthal direction such that radiation is received earlier than if there were no rotation.

If $\zeta$ is the angle between the rotation axis and the line-of-sight then the distance from the rotation axis to the line-of-sight is $r_{\mathrm{em}}^{\mathrm{i}} \sin \zeta$. Hence the rotation velocity at the emission point is $\Omega r_{\mathrm{em}}^{\mathrm{i}} \sin \zeta$. If $v_{\|}$is the velocity of particles along the field line then the total velocity of particles $\mathbf{v}_{\text {tot }}=\hat{b} v_{\|}+\hat{\phi} \Omega r_{\mathrm{em}}^{\mathrm{i}} \sin \zeta$, where $\hat{b}$ and $\hat{\phi}$ are the unit vectors in the directions of magnetic field and rotation, respectively. The aberration angle $\eta_{\mathrm{ab}}^{\mathrm{i}}$ is given by

$$
\sin \eta_{\mathrm{ab}}^{\mathrm{i}}=\frac{\Omega r_{\mathrm{em}}^{\mathrm{i}} \sin \zeta}{v_{\mathrm{tot}}},
$$

where $v_{\text {tot }}=\left|\mathbf{v}_{\text {tot }}\right|$. It is more appropriate to assume $v_{\text {tot }} \approx c$ instead of $v_{\|} \approx c$, therefore, we have

$$
\sin \eta_{\mathrm{ab}}^{\mathrm{i}}=\frac{\Omega r_{\mathrm{em}}^{\mathrm{i}} \sin \zeta}{c}=\frac{r_{\mathrm{em}}^{\mathrm{i}}}{r_{\mathrm{LC}}} \sin \zeta
$$

where $r_{\mathrm{LC}}=c / \Omega$ is the radius of the velocity of light cylinder for the pulsar. Hence the aberration is greater for emissions arising at larger altitudes.

Our aberration angle formula (Eq. 6) differs in two respects with respect to the one given in the literature (e.g., Cordes 1978; Phillips 1992): (i) literature formula uses $\alpha$ instead of $\zeta$, which is correct only when the magnetic axis and the line-of-sight are aligned $(\beta=0)$, and (ii) we estimate ' $\sin \eta_{\mathrm{ab}}^{\mathrm{i}}$ ' instead of ' $\tan \eta_{\mathrm{ab}}^{\mathrm{i}}$ ' as it avoids $v_{\text {tot }}$ exceeding c when we set $v_{\|} \approx c$. However, the values of aberration angle estimated using the two formulae differ by a very little, i.e., of order $\left(r_{\mathrm{em}}^{\mathrm{i}} / r_{\mathrm{LC}}\right)^{2}$ for $\beta \ll \alpha$.

If the cones are emitted at different altitudes from the surface of the neutron star then the conal radiation emitted at lower altitudes takes more time to reach the observer compared to the arrival times of those components emitted at higher altitudes. In terms of pulse phase of the received radiation, this is equivalent to a shift of components emitted at higher altitude to earlier phase with respect to phases of lower altitude components. If $r_{\mathrm{em}}^{\mathrm{i}}$ is the emission altitude of $i^{\text {th }}$ cone, then this retardation phase shift is given by

$$
\eta_{\mathrm{ret}}^{\mathrm{i}}=\frac{r_{\mathrm{em}}^{\mathrm{i}}}{r_{\mathrm{LC}}}
$$


The net phase shift due to aberration and retardation is $\eta^{\mathrm{i}}=\eta_{\mathrm{ab}}^{\mathrm{i}}+\eta_{\mathrm{ret}}^{\mathrm{i}}$, which gives

$$
\sin \left(\eta^{\mathrm{i}}-\frac{r_{\mathrm{em}}^{\mathrm{i}}}{r_{\mathrm{LC}}}\right)=\frac{r_{\mathrm{em}}^{\mathrm{i}}}{r_{\mathrm{LC}}} \sin \zeta .
$$

For the small angle approximation $\left(\eta_{\mathrm{ab}}^{\mathrm{i}} \ll 1\right)$, the solution of this equation is given by

$$
r_{\mathrm{em}}^{\mathrm{i}} \approx \frac{r_{\mathrm{LC}} \eta^{\mathrm{i}}}{(1+\sin \zeta)} .
$$

Both aberration and retardation shift the pulse components emitted at higher altitudes to earlier phases in the received pulse. Therefore, we see an asymmetry in the location of the leading and trailing components with respect to the centre of the profile. If $\phi_{1}^{\mathrm{i}}$ and $\phi_{\mathrm{t}}^{\mathrm{i}}$ are the measured locations of the leading and trailing components of a cone with respect to the centre of the profile, then

$$
\phi_{1}^{\mathrm{i}}=-\phi^{\mathrm{i}}+\eta^{\mathrm{i}} ; \quad \phi_{\mathrm{t}}^{\mathrm{i}}=\phi^{\mathrm{i}}+\eta^{\mathrm{i}},
$$

where $\phi^{\mathrm{i}}$ is the phase of $\mathrm{i}^{\text {th }}$ component when the aberration and retardation are absent. From these, the values of $\eta^{\mathrm{i}}$ and $\phi^{\mathrm{i}}$ are easily obtained :

$$
\eta^{\mathrm{i}}=\frac{\left(\phi_{\mathrm{t}}^{\mathrm{i}}+\phi_{1}^{\mathrm{i}}\right)}{2} ; \quad \phi^{\mathrm{i}}=\frac{\left(\phi_{\mathrm{t}}^{\mathrm{i}}-\phi_{1}^{\mathrm{i}}\right)}{2} .
$$

The estimate of the net phase shift $\eta^{\mathrm{i}}$, in conjunction with Eq. (8), immediately yields an estimate for the emission height $r_{\mathrm{em}}^{\mathrm{i}}$ of the $i^{\text {th }}$ cone. Next, using the phase locations $\phi^{\mathrm{i}}$ of the conal components, we can estimate the tangent angle $\Gamma^{\mathrm{i}}$ from Eq. (3), and the angular location $\theta_{\mathrm{em}}^{\mathrm{i}}$ of the emission point from Eq. (4).

Further, the knowledge of emission height and angular location can be used to map the respective locations of the "foot" points of field lines associated with the emission region of each cone on the polar cap. For this, we start with the equation of dipolar field lines

$$
r=r_{\mathrm{e}} \sin ^{2} \theta,
$$

where $r_{\mathrm{e}}$ is the field line constant, which is the equatorial distance of a field line from magnetic axis. Applying this at the surface of the neutron star gives

$$
\frac{1}{r_{\mathrm{e}}^{\mathrm{i}}}=\frac{\sin ^{2} \theta_{s}^{\mathrm{i}}}{r_{\mathrm{s}}}=\frac{\sin ^{2}\left(s^{\mathrm{i}} / r_{\mathrm{s}}\right)}{r_{\mathrm{s}}} \simeq \frac{s^{\mathrm{i}^{2}}}{r_{\mathrm{s}}^{3}},
$$

where $s^{\mathrm{i}}$ is the distance from the magnetic axis to the foot of the field line (colatitude), as measured on the surface of neutron star with radius $r_{\mathrm{s}}(\sim 10 \mathrm{~km})$. The approximate version 
in the above equation is for the case $\theta_{\mathrm{s}}^{\mathrm{i}} \ll 1$. At the emission point, the dipole field equation then yields

$$
\frac{\sin ^{2}\left(\theta_{\mathrm{em}}^{\mathrm{i}}\right)}{r_{\mathrm{em}}^{\mathrm{i}}}=\frac{\sin ^{2}\left(s^{\mathrm{i}} / r_{\mathrm{s}}\right)}{r_{\mathrm{s}}} .
$$

Under the small angle approximation we obtain the following relationship :

$$
s^{\mathrm{i}} \simeq s_{\mathrm{L}} \sqrt{\frac{r_{\mathrm{LC}}}{r_{\mathrm{em}}^{\mathrm{i}}}} \sin \theta_{\mathrm{em}}^{\mathrm{i}} .
$$

Here $s_{\mathrm{L}} \approx\left(r_{\mathrm{s}}^{3} / r_{\mathrm{LC}}\right)^{1 / 2}$ is the distance from the magnetic axis to the foot of the last open field line that demarcates the polar cap region.

Using the above formulae, we have computed, for PSR B0329+54, the emission heights and the location of the relevant field lines, independently for each of the four cones and at each of the two frequencies. We have used $\alpha=30^{\circ}$ and $\beta=2.1^{\circ}$, as proposed by Rankin (1993) for this pulsar. Further, we have taken the fiducial centre point of the profile as the location of the core component, assuming that it is emitted at a very small height above the neutron star surface. Our results are summarized in Table 2 for the lower frequency $(325 \mathrm{MHz})$ and in Table 3 for the higher frequency $(606 \mathrm{MHz})$. The second and third columns give the measured locations of the leading and trailing components for each cone (with respect to the location of the core component), and the next two columns give the inferred values for net phase shifts and tangent angles for the leading component of each cone. Column 6 gives the estimates of emission height (in $\mathrm{km}$ ) for each cone. Column 7 gives the estimates for the transverse location of the associated field lines on the polar cap. This is characterized by $s_{\mathrm{L}}^{\mathrm{i}}=s^{\mathrm{i}} / s_{\mathrm{L}}$. In the case of PSR B0329+54, the magnetic and rotation axes are inclined through an angle $\alpha=30^{\circ}$, therefore, the polar cap is actually ellipsoidal with major axis aligned in the longitudinal direction of the pulsar (Cordes 1978). For the normalizing purpose we consider the position of the last open filed line which is on the azimuthal direction, $s_{\mathrm{L}} \approx\left(r_{\mathrm{s}}^{3} / r_{\mathrm{LC}}\right)^{1 / 2} \sim 171 \mathrm{~m}$. The errors in component location are propagated appropriately to estimate the errors for all other dependent parameters given in Table 2 and Table 3.

The salient results from our interpretation of the emission geometry are as follows :

(i) For the same cone, the lower frequency radio radiation is emitted at a higher altitude than the higher frequency radiation. This result supports the canonical picture of radius-tofrequency mapping.

(ii) For the same frequency, successive cones are emitted at higher altitudes in the pulsar magnetosphere. The heights range from a nearly a hundred kilometers for the innermost cone 
to a thousand kilometers for the outermost cone or from about $0.5 \%$ to about $3 \%$ of the light cylinder radius.

(iii) All cones, at both frequencies appear to originate on (or around) a narrow set of field lines, slightly more than half-way out to the edge of the polar cap region $\left(\mathrm{s}^{\mathrm{i}} / \mathrm{s}_{\mathrm{L}} \approx 0.5-0.6\right)$.

\subsection{Effect of magnetic field sweepback}

We now consider the effect of magnetic field sweepback, an effect that opposes the aberration effect. Since pulsars lose their rotation energy by magnetic dipole radiation, the magnetic dipole field experiences a torque due to such emissions. As a result, the field lines tend to bend in the toroidal directions, which is opposite to the sense of rotation. To estimate the bending angle at the emission altitude $r_{\mathrm{em}}^{\mathrm{i}}$ we use the following result given by Shitov (1983)

$$
\phi_{\mathrm{mfs}}^{\mathrm{i}}=1.2\left(\frac{r_{\mathrm{em}}^{\mathrm{i}}}{r_{\mathrm{LC}}}\right)^{3} \sin ^{2} \alpha
$$

Using the emission altitudes given in the Table 2, we computed the bending angle due to field sweepback for each cone, at each frequency, and found the sweepback is too small compared to the aberration and retardation phase shifts. For the outer most cone, where the sweepback is expected be highest, we find $\phi_{\mathrm{mfs}}^{\mathrm{i}}<0.001^{\circ}$ at $325 \mathrm{MHz}$ and $0.0005^{\circ}$ for 610 $\mathrm{MHz}$. Therefore, our assumption that the phase shifts of the cone centers with respect to the core position are predominantly due to the aberration and retardation, is validated.

Table 2: Observed and inferred locations of conal components at $325 \mathrm{MHz}$.

\begin{tabular}{ccccccc}
\hline \hline $\begin{array}{c}\text { Cone } \\
\text { No. }\end{array}$ & \multicolumn{1}{c}{$\begin{array}{c}\phi_{\mathrm{l}}^{\mathrm{i}} \\
(\mathrm{deg})\end{array}$} & $\begin{array}{c}\phi_{\mathrm{t}}^{\mathrm{i}} \\
(\mathrm{deg})\end{array}$ & $\begin{array}{c}\eta^{\mathrm{i}} \\
(\mathrm{deg})\end{array}$ & $\begin{array}{c}\Gamma^{\mathrm{i}} \\
(\mathrm{deg})\end{array}$ & $\begin{array}{c}r_{\mathrm{em}}^{\mathrm{i}} \\
(\mathrm{km})\end{array}$ & $s_{\mathrm{L}}^{\mathrm{i}}$ \\
\hline 1 & $-5.5 \pm 0.44$ & $4.5 \pm 0.29$ & $-0.5 \pm 0.26$ & $3.3 \pm 0.11$ & $180 \pm 100$ & $0.5 \pm 0.16$ \\
2 & $-9.5 \pm 0.21$ & $7.3 \pm 0.31$ & $-1.1 \pm 0.19$ & $4.8 \pm 0.09$ & $430 \pm 070$ & $0.5 \pm 0.04$ \\
3 & $-14.0 \pm 0.34$ & $10.0 \pm 0.42$ & $-2.0 \pm 0.27$ & $6.5 \pm 0.13$ & $770 \pm 110$ & $0.5 \pm 0.04$ \\
4 & $-20.0 \pm 1.43$ & $14.1 \pm 0.72$ & $-3.0 \pm 0.80$ & $9.0 \pm 0.40$ & $1150 \pm 310$ & $0.6 \pm 0.08$ \\
\hline
\end{tabular}




\section{Discussion}

Our results show that PSR B0329+54 has 9 unique emission components (of which at least 3 have been detected for the first time). This is the largest number of components detected for any pulsar and indicates that the emission geometry for this pulsar is probably quite complicated. We note that our component VI, detected in the longitude range of $-8^{\circ}$ to $-10^{\circ}$, matches very well with the sixth component proposed by Kuzmin and Izvekova (1996) as a result of Gaussian fits to the emission components. Our results thus confirm the component proposed by them.

The new components are clearly seen at $606 \mathrm{MHz}$ but are less prominent at $325 \mathrm{MHz}$. This may be part of the known trend of reducing significance of conal components (with respect to the core component) at lower frequencies. Further, the locations of components at the two frequencies follow the commonly seen trend that distance from the centre of the profile increases at lower frequencies, generally referred to as the "radius-to-frequencymapping" effect, (e.g. Phillips 1992; Sieber 1996). This also supports the argument of genuineness of the detected components. Our results also appear to indicate that the newly detected conal components for this pulsar show up more clearly during the abnormal mode rather than in the normal mode.

Our interpretation of the 9 emission components as 4 nested cones of emission surrounding a single core component supports the picture of core and conal emission beams (e.g. Rankin 1983a; Oster and Sieber 1977). Rankin (1993) has proposed 2 conal rings to explain the existence of pulsars with 5 components in their average profiles. More recently, Mitra and Deshpande (1999) have found evidence for 3 nested conal rings, from their analysis of the emission patterns of a large sample of pulsars. PSR B0329+54 is the first pulsar for which we found the clear evidence for as many as 4 nested cones of emission. It should be interesting to see if a similar analysis for other multicomponent pulsar shows the presence of 4 emission cones.

Table 3: Observed and inferred locations of conal components at $606 \mathrm{MHz}$.

\begin{tabular}{ccrcccc}
\hline \hline $\begin{array}{c}\text { Cone } \\
\text { No. }\end{array}$ & \multicolumn{1}{c}{$\begin{array}{c}\phi_{1}^{\mathrm{i}} \\
(\mathrm{deg})\end{array}$} & $\begin{array}{c}\phi_{\mathrm{t}}^{\mathrm{i}} \\
(\mathrm{deg})\end{array}$ & $\begin{array}{c}\eta^{\mathrm{i}} \\
(\mathrm{deg})\end{array}$ & $\begin{array}{c}\Gamma^{\mathrm{i}} \\
(\mathrm{deg})\end{array}$ & $\begin{array}{c}r_{\mathrm{em}}^{\mathrm{i}} \\
(\mathrm{km})\end{array}$ & $s_{\mathrm{L}}^{\mathrm{i}}$ \\
\hline 1 & $-5.3 \pm 0.35$ & $4.5 \pm 0.37$ & $-0.4 \pm 0.25$ & $3.3 \pm 0.10$ & $160 \pm 100$ & $0.6 \pm 0.17$ \\
2 & $-8.8 \pm 0.34$ & $6.9 \pm 0.14$ & $-1.0 \pm 0.18$ & $4.6 \pm 0.08$ & $380 \pm 070$ & $0.5 \pm 0.05$ \\
3 & $-12.6 \pm 0.64$ & $9.5 \pm 0.64$ & $-1.6 \pm 0.46$ & $6.0 \pm 0.22$ & $600 \pm 180$ & $0.5 \pm 0.08$ \\
4 & $-17.9 \pm 0.79$ & $13.6 \pm 0.88$ & $-2.2 \pm 0.59$ & $8.4 \pm 0.29$ & $840 \pm 230$ & $0.6 \pm 0.09$ \\
\hline
\end{tabular}


Our data also presents the direct evidence for the detection of aberration and retardation effects in pulsar magnetospheres. Earlier experimental efforts to detect evidence for aberration and retardation time delays (as well as magnetic field sweepback) in some pulsars (e.g. Phillips 1992) did not yield any positive signature. Recently, Malov and Suleimanova (1998) have estimated the aberration and retardation phase shifts for components I and IV of this pulsar using the average pulse profiles at different frequencies. The detection of aberration and retardation phase shifts of as much as three degrees that we report, supports the view that the conal emission originates at altitudes of the order of several hundreds of kilometers above the neutron star surface. Rankin (1993) reports an emission altitude of $217 \mathrm{~km}$ at $1 \mathrm{GHz}$ for the main conal ring (cone number 3 in our picture) of this pulsar. Kijak and Gil $(1997,1998)$ estimate somewhat higher emission altitudes, in the range $300-500$ $\mathrm{km}$. From our results, the corresponding emission altitude is about $600 \mathrm{~km}$ at $606 \mathrm{MHz}$.

Further, the fact that a given conal ring exhibits more aberration at the lower frequency clearly shows that the lower frequency radiation originates at a higher altitude in the magnetosphere of this pulsar. This radius-to-frequency-mapping effect has been proposed as an explanation for the commonly observed fact that the overall widths of pulsar profiles increase with decreasing frequency. Our results provide a direct corroboration of this model, and the result then makes other proposed explanations, such as chromatic aberration (or refraction) in the magnetosphere (e.g. Barnard and Arnos 1986) or low frequency broadening due to reduced beaming of the radiating particles (Kunzl et al. 1998), less likely to be valid.

By combining the emission heights obtained from aberration and retardation effects with a dipolar magnetic field geometry, we have been able to localize the exact emission point of each cone with a good degree of precision. Perhaps the most interesting result of our work is the conclusion that the successive cones of emission originate at increasing heights in the magnetosphere, but along relatively nearby field lines. This is for the first time that such a result has been obtained for any pulsar. In the context of 2 cones of emission, Rankin (1993) has raised this as a major unanswered question: whether the cones are emitted at different heights along the same set of field lines or are associated with different sets of field lines. For PSR B0329+54, this question is now answered for all the 4 cones of emission. We find that all the cones at a given frequency are associated with a set of field lines that are located at about $0.5-0.6$ of the distance to the edge of the polar cap. We believe that the scatter in the values ( 0.5 to 0.6 ) for different cones is within the limits of our estimation errors. Further, we find that the cones at both frequencies are also associated with the same set of field lines, again within the error estimates.

We note in particular that our results show clearly that the radio emission does not originate at or near the last open field line region of pulsar magnetosphere. In the formulation 
we have presented in Eqs. (1) through (15), there is only one free parameter that can affect this conclusion: the value of $\alpha$, the inclination angle between the magnetic and rotation axes of the pulsar. Higher values of $\alpha$ would reduce the estimated emission heights and consequently move the associated field lines closer to the edge of the polar cap. However, there good evidence that the value of $\alpha=30^{\circ}$ for this pulsar is a robust estimate (Rankin 1990; Lyne and Manchester 1988).

If the results about the emission geometry that we have obtained for pulsar B0329+54 are found to be true in general for the population of known pulsars, then it should prove to be a significant improvement in our general understanding of the emission geometry of radio pulsars. This could provide important constraints for the various theories for the emission mechanism of radio pulsars. For example, any successful theory would need to produce radiation at a given frequency from significantly different heights along the same set of field lines in the magnetosphere to explain the observed behavior of conal components.

\section{Conclusion}

We have used a technique based on windowing and thresholding, to detect the weak emission components in pulsar profiles. By applying this to the single pulse data of PSR B0329+54 at $325 \mathrm{MHz}$ and $606 \mathrm{MHz}$, we have detected three new emission components for this pulsar, and also confirmed the presence of a new component proposed by Kuzmin and Izvekova (1996). We conclude that this pulsar has 9 components, which is the highest among all the known pulsars.

We interpret the 9 components as being produced by 4 nested hollow cones of emission along with a central core component of emission. Our results thus support the core and conal emission picture of pulsar radiation beams.

We find clear evidence that the conal components in the trailing part of the pulsar profile are squeezed closer to the core (and to each other) as compared to the corresponding components in the leading part of the profile. We interpret this as evidence for aberration and retardation effects in the conal beams. The observed aberration and retardation phase shifts increase from the inner to outer cones, and for any given cone, the effect is more at the lower frequency.

From a detailed consideration of the emission geometry in the magnetosphere, including the effects of aberration and retardation, we are able to constrain the location of emission regions independently for each cone, and at each frequency $(325 \& 606 \mathrm{MHz})$. From these computations, we are able to conclude the following: (i) for the same cone, the lower fre- 
quency radiation comes from a higher altitude than the higher frequency radiation, (ii) for the same frequency, successive outer cones originate at higher altitudes and (iii) all cones at both frequencies appear to originate on (or around) a specific set of field lines which are located at about half-way out to the edge of the polar cap region. The inferred emission heights for the cones range from $\sim 160 \mathrm{~km}$ to $\sim 1150 \mathrm{~km}$.

It is, probably, for the first time that the emission regions corresponding to the conal components for a pulsar have been located so precisely and unambiguously. In particular, our conclusion that there is only a restricted region of the polar cap that is active in producing all the conal radiation that is observed, should prove to be an interesting constrain for models or theories of pulsar emission mechanisms.

We thank Dunc Lorimer for his help in data reduction and fruitful discussions during the initial stages of the work. We are thankful to A. G. Lyne for providing the Jodrell Bank data and to J. M. Rankin, A. Peyman and D. Mitra for useful discussions.

\section{REFERENCES}

Barnard, J. J., \& Arons, J. 1986, ApJ, 302, 138

Cordes, J. M. 1978, ApJ, 222, 1006

Gangadhara, R. T. 2000, BASI, 28, 297

Gil, J. A. 1991, A\&A, 243, 219

Gould, D. M., \& Lyne, A. G. 1998, MNRAS, 301, 235

Gupta, Y., Gothoskar, P. G., Joshi, B. C., Vivekanand, M., Swain, R., Sirothia, S., \& Bhat, N. D. R., 2000, in IAU Colloquium 177: Pulsar Astronomy - 2000 and Beyond, eds. M. Kramer, N. Wex \& R. Wielebinski, ASP Conf. Series, 105, 277

Hesse, K. H. 1973, A\&A27, 373

Hesse, K. H., Sieber, W., \& Wielebinski, R. 1973, Nature, 245, 57

Kijak, J., \& Gil, J. 1997, MNRAS, 288, 631

Kijak, J., \& Gil, J. 1998, MNRAS, 299, 855

Kunzl, T., Lesch, H., Jessner, A., \& von Hoensbroech, A. 1998, ApJ, 505, L139 
Kuzmin, A. D., \& Izvekova, V. A. 1996, IAU Colloquium 160: Pulsars: Problems \& Progress, eds. S. Johnston, M.A. Walker \& M. Bailes, ASP Conf. Seri. 105, 217

Lyne, A. G. 1971, MNRAS, 153, 27P

Lyne, A. G., \& Manchester, R. N. 1988, MNRAS, 234, 477

Malov, I. F., \& Suleimanova, S. A. 1998, Astron. Rep., 42, 388

Michel, F. C. 1991, Theory of Neutron Star Magnetospheres, Univ. of Chicago Press, Chicago, p. 114

Mitra, D., \& Deshpande, A. A. 1999, A\&A, 346, 906

Phillips, J. A. 1992, ApJ, 385, 282

Oster, L., \& Sieber, W. 1977, ApJ, 58, 303

Rankin, J. M. 1983a, ApJ, 274, 333

Rankin, J. M. 1983b, ApJ, 274, 359

Rankin, J. M. 1990, ApJ, 352, 247

Rankin, J. M. 1993, ApJS, 85, 145

Sieber, W. 1996, IAU Colloquium 160: Pulsars: Problems \& Progress, eds. S. Johnston, M.A. Walker \& M. Bailes, ASP Conf. Seri. 105, 167

Shitov, Yu. P. 1983, Sov. Astron. 27, 314

Swarup, G., Ananthakrishnan, S., Subrahmanya, C. R., Rao, A. P., Kulkarni, V. K., \& Kapahi, V. K. 1990, in High Sensitivity Radio Astronomy, ed. N. Jackson \& R.J. Davis (Cambridge Univ. Press) 

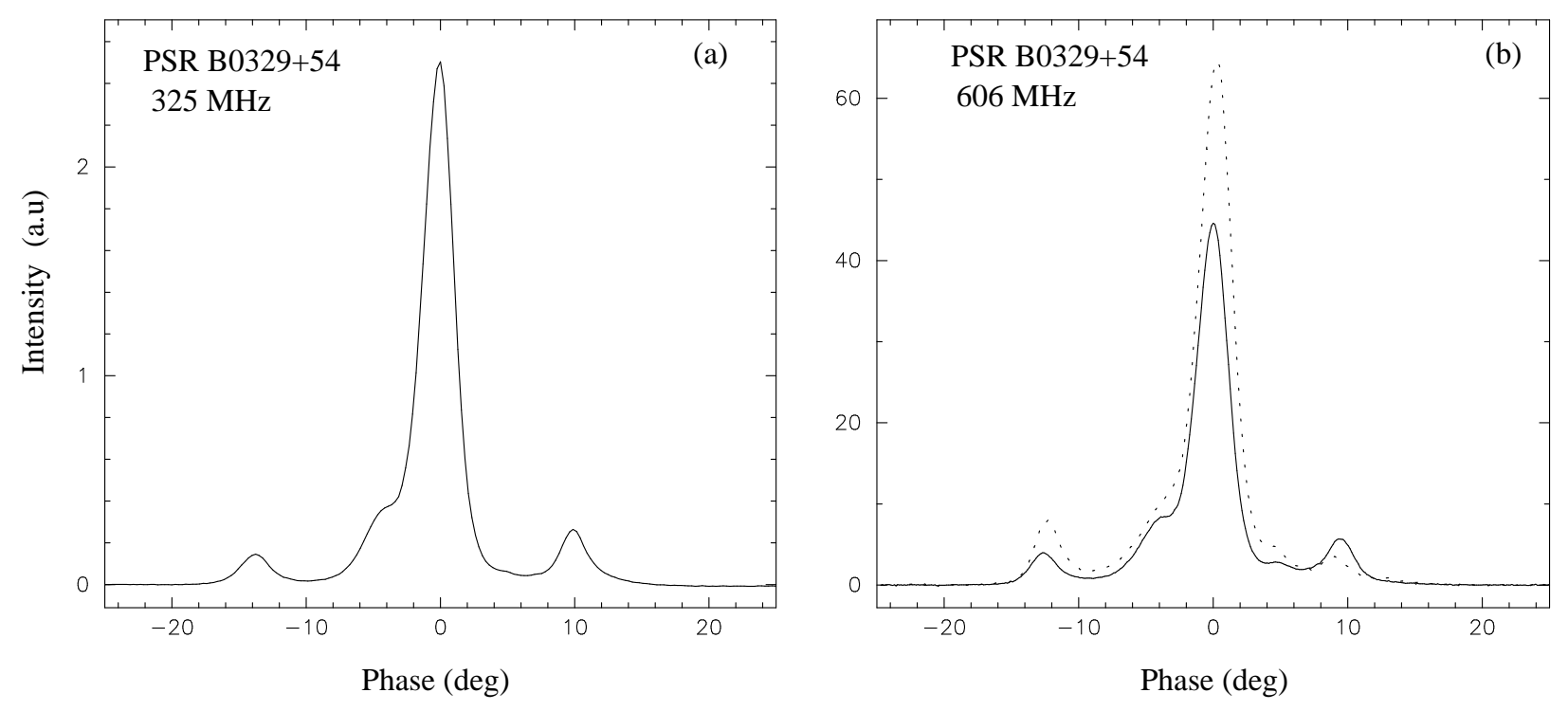

Fig. 1.- Average pulse profiles for PSR B0329+54 obtained from the data at $325 \mathrm{MHz}$ and $606 \mathrm{MHz}$. The intensity is in arbitrary units. For the $606 \mathrm{MHz}$ data, the dotted line curve shows the profile for the abnormal mode of the pulsar. 

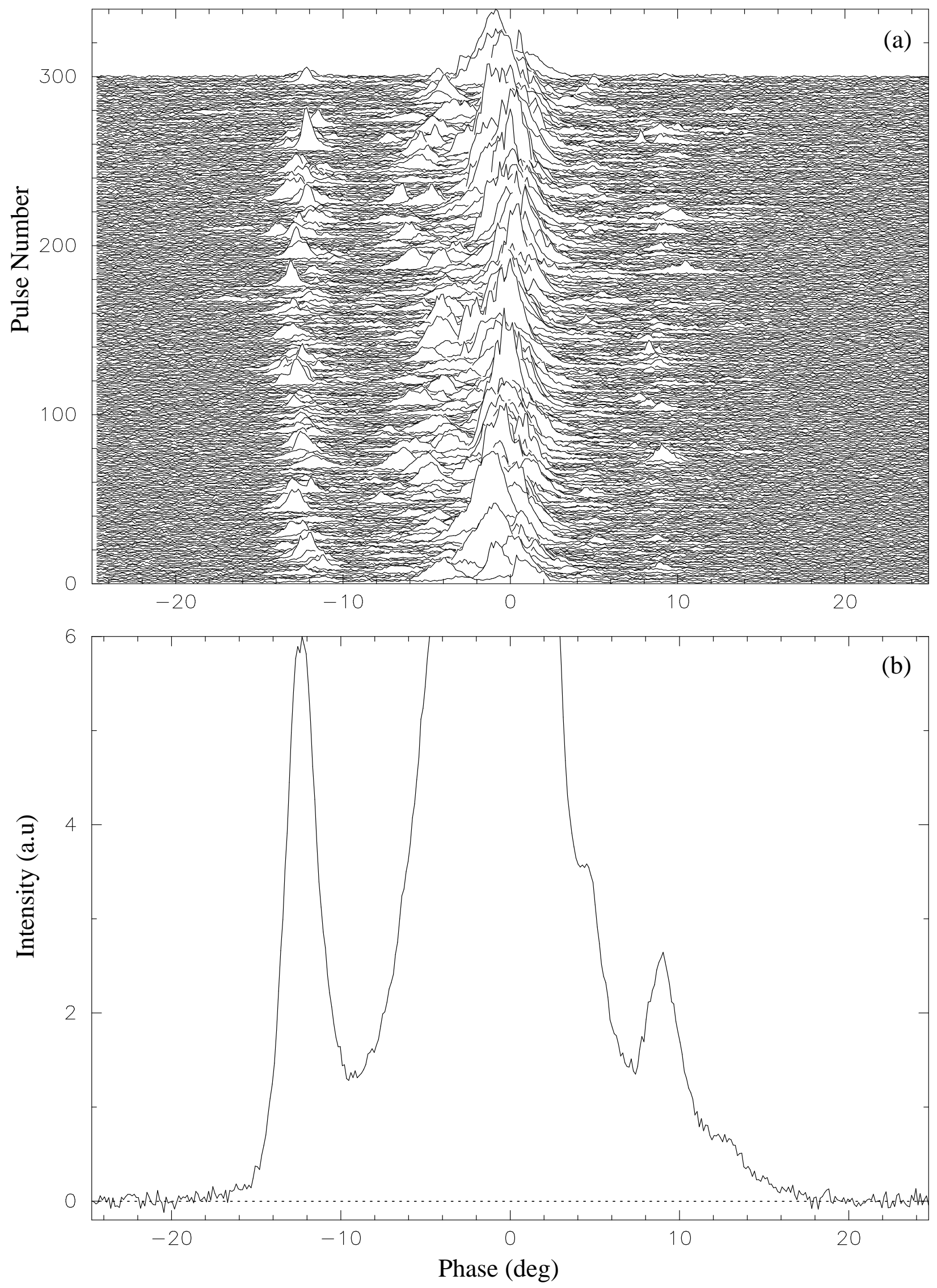

Fig. 2.- (a) Sequence of single pulses from PSR B0329+54 at $606 \mathrm{MHz}$, and (b) an average 

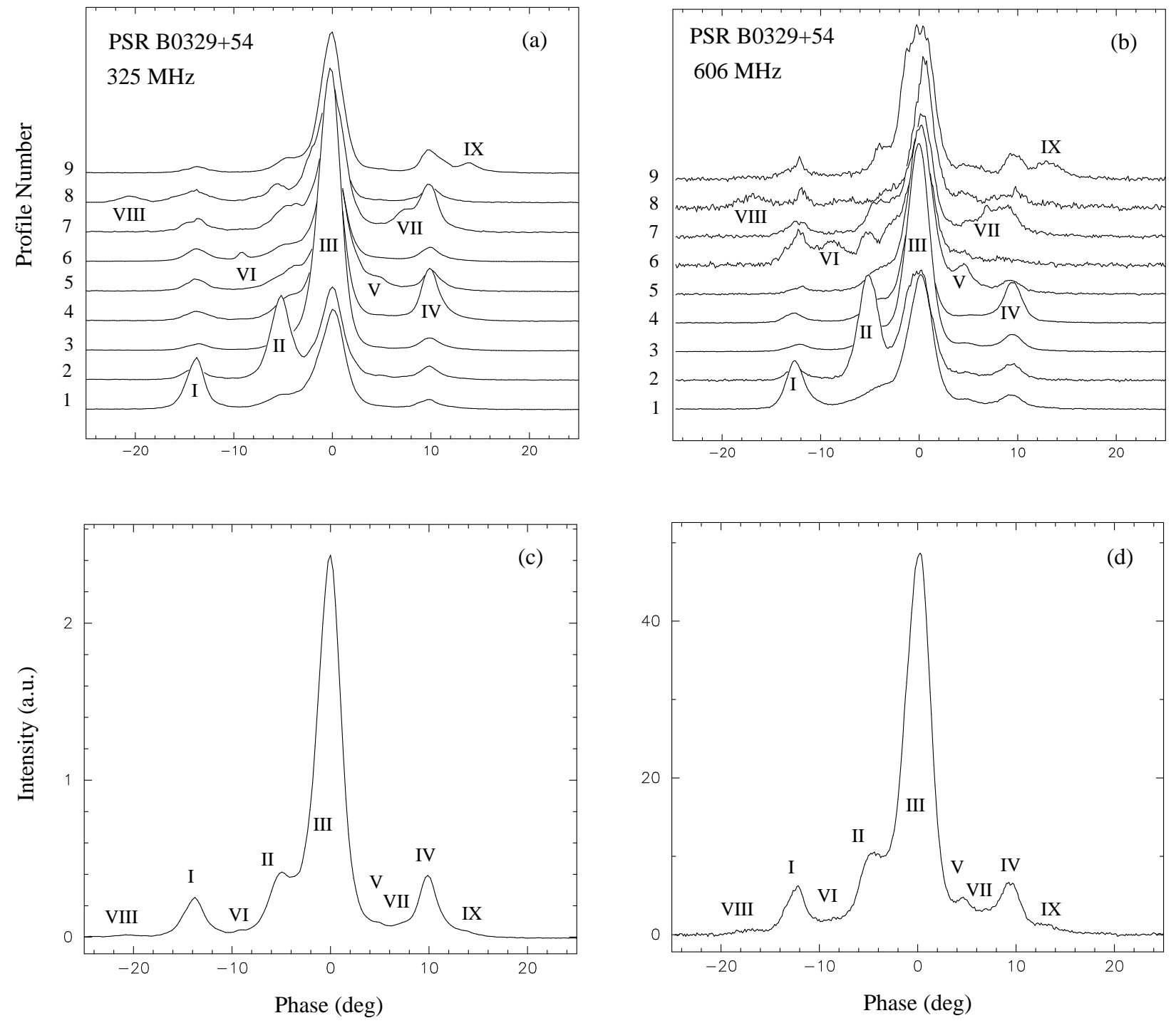

Fig. 3.- (a)\&(b) show the average profiles (in arbitrary units) obtained for each of the nine detected components by using the window-threshold technique at $325 \mathrm{MHz}$ and $606 \mathrm{MHz}$ while $(\mathrm{c}) \&(\mathrm{~d})$ show the averages of these nine profiles. 


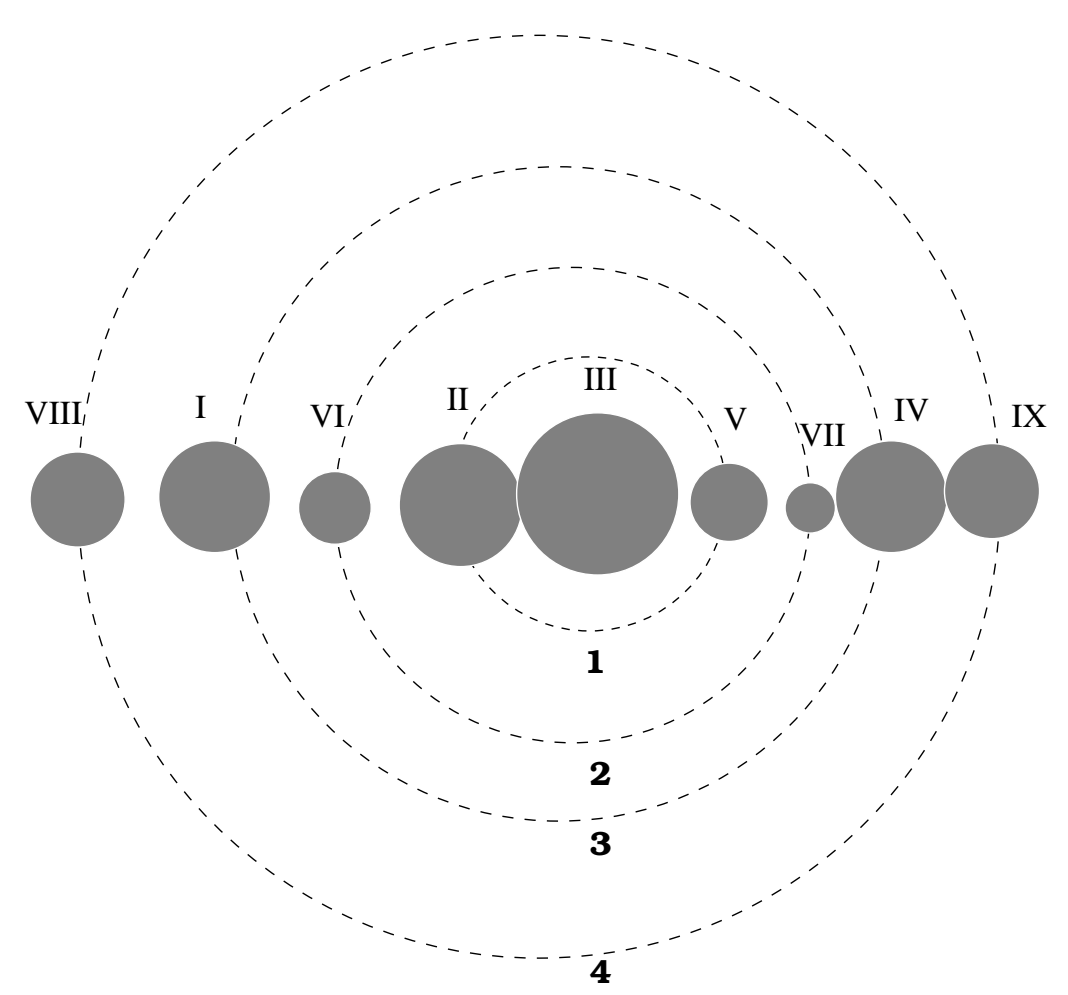

(a)

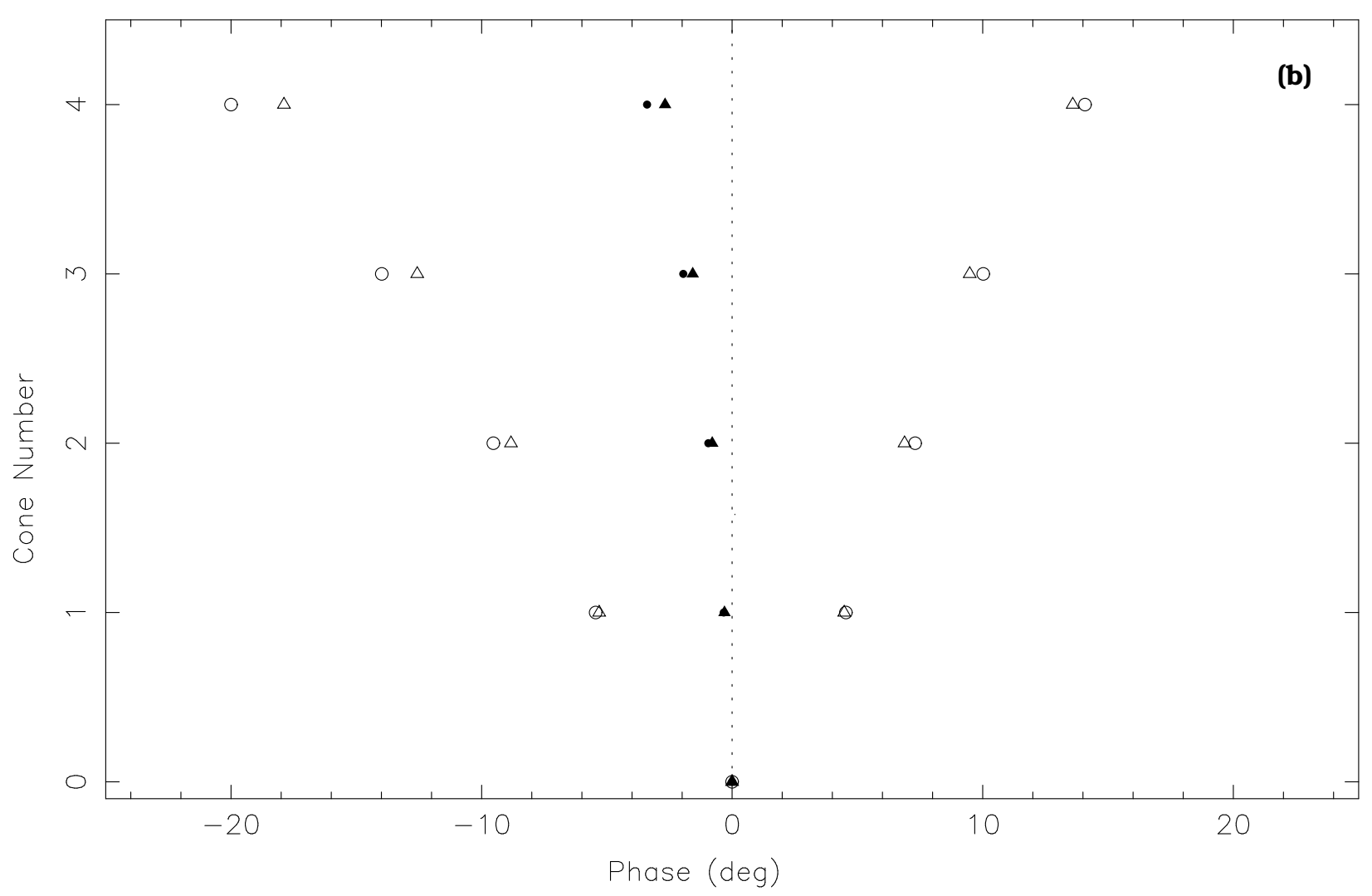

Fig. 4.- (a) Location of the nine emission components at $606 \mathrm{MHz}$, shown in the form of 4 conal rings around the central core component, and (b) extent (open symbols) and centre (filled symbols) of the cones at $325 \mathrm{MHz}$ (circles) and $606 \mathrm{MHz}$ (triangles). The core 


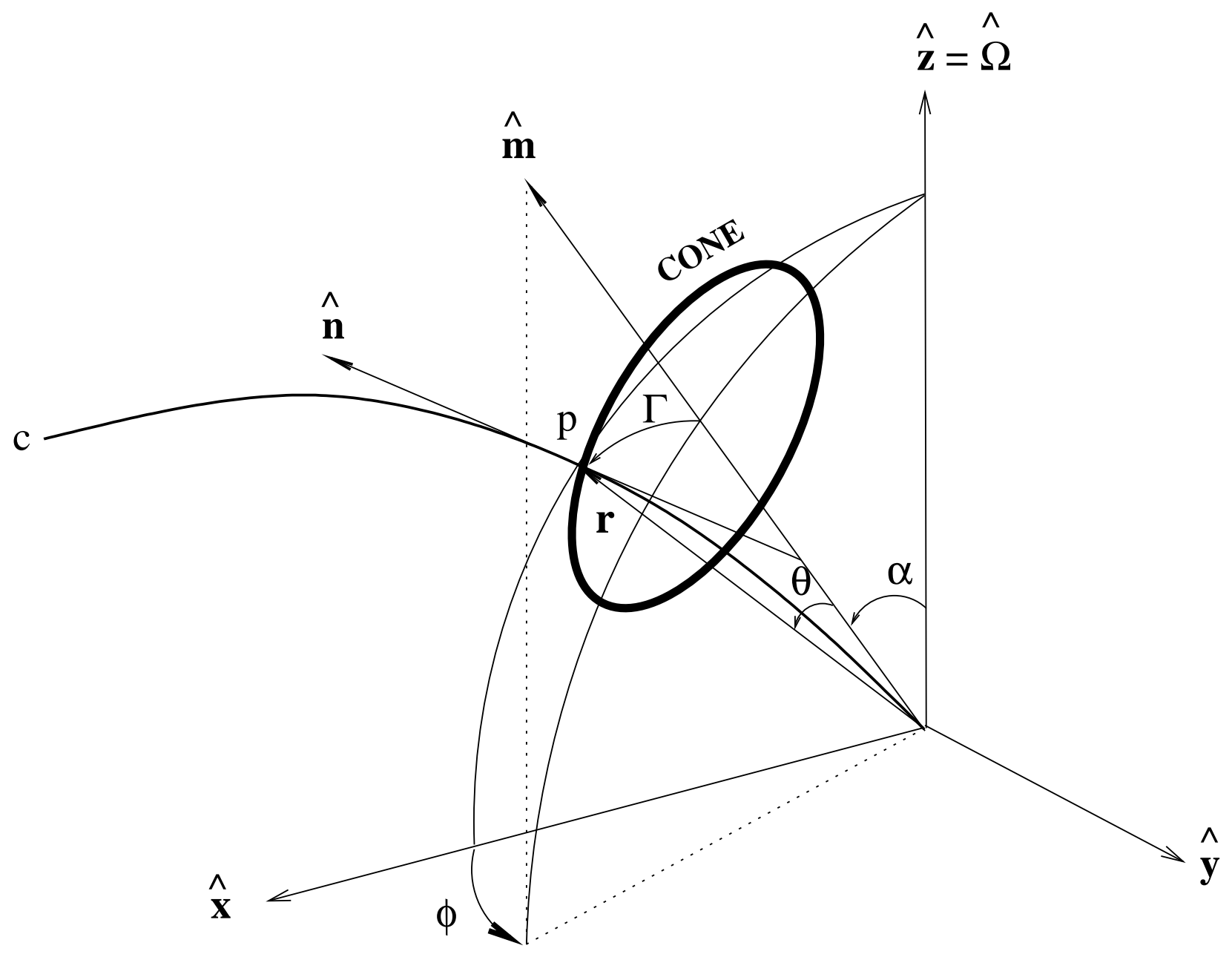

Fig. 5.- Emission geometry, where $\hat{n}$ is tangent to the dipolar field line $\mathrm{C}$ and directing towards the observer's line-of-sight, $\mathbf{r}$ is the position vector of emission point $\mathrm{p}, \hat{m}$ is the magnetic axis, and $\phi$ is the pulse phase. 